\title{
État civil et suicide des Bertillon à Durkheim
}

\section{Massimo Borlandi}

\section{OpenEdition}

\section{Journals}

Édition électronique

URL : http://journals.openedition.org/ress/359

DOI : $10.4000 /$ ress.359

ISSN : 1663-4446

\section{Éditeur}

Librairie Droz

\section{Édition imprimée}

Date de publication : 1 mars 2004

Pagination : 23-37

ISBN : 2-600-00941-8

ISSN : 0048-8046

Référence électronique

Massimo Borlandi, «État civil et suicide des Bertillon à Durkheim », Revue européenne des sciences sociales [En ligne], XLII-129 | 2004, mis en ligne le 04 novembre 2009, consulté le 20 avril 2019. URL http://journals.openedition.org/ress/359; DOI : 10.4000/ress.359 
Massimo BORLANDI

\section{ÉTAT CIVIL ET SUICIDE DES BERTILLON À DURKHEIM}

L'étude empirique du bonheur des Français ne connaît de véritables progrès au $\mathrm{XIX}^{\mathrm{e}}$ siècle qu'à la suite de la publication, dans le Compte général de l'administration de la justice criminelle, de l'état civil des suicidés selon le sexe et le fait qu'ils aient ou non des enfants. Et ce à partir du volume de l'année 1863, paru en 1865. Il devient ainsi possible de déterminer, de même que le taux de suicide des célibataires et celui des époux et des veufs (combien sur un million d'individus de même condition?), la tendance au suicide de chacun de ces trois groupes relativement à (ou en fonction de) celle des deux autres. On fait d'ordinaire ce calcul en mettant en rapport les taux de suicide de la façon suivante: pour 100 époux qui se donnent la mort, combien de célibataires et combien de veufs accomplissent le même geste? et pour 100 veufs, combien de célibataires? Il s'agit toutefois d'évaluations imparfaites et destinées à le rester car le Compte donne l'état civil des suicidés indépendamment de l'âge et continuera à le faire.

Il demeure par contre impossible d'établir correctement dans quelle mesure la présence d'enfants protège du suicide, du moins jusqu'au recensement de la population de 1886 qui fait connaître, en 1888, le nombre des époux et des veufs avec et sans enfants, condition indispensable pour qu'on puisse calculer les taux de suicide des pères et des mères. Avant cette date - hormis le seul cas dont je parlerai - on se rabat sur des proportions. Encore faut-il préciser qu'on ne disposera jamais, dans les dénombrements, de la distribution selon l'âge des ménages avec et sans enfants.

Ces limites étant posées, le premier à mesurer, à la fois, le taux des trois catégories d'état civil et leur tendance relative au suicide est Louis-Adolphe Bertillon, dans l'article «Mariage» (1872) du Dictionnaire encyclopédique des sciences médicales d'Amédée Dechambre. Il donne aussi la première estimation de l'effet de la présence d'enfants sur l'aptitude au suicide des époux basée sur la proportion des pères et des mères sur le total de suicidés de ces deux groupes. Bertillon lui-même et son fils Jacques intègrent à cet article des compléments substantiels en 1879 et 1880 .

Avant les travaux des Bertillon, la page sur les relations entre état civil et suicide est la plus vide de l'histoire - prometteuse - de la statistique appliquée aux événements moraux de l'Hexagone. Adolphe Quetelet $(1835,2,159)$ doit se contenter de ce qu'il trouve dans un rapport sur un livre de Jean-Pierre Falret (1822) lu à l'Institut, à savoir que «pour les hommes, ce sont les célibataires qui donnent le chiffre le plus élevé, et pour les femmes, on le trouve parmi celles qui sont engagées dans les liens du mariage ». Rien ne semble justifier les impressions (annonçant toutefois des thèses bien fondées) que ce rapporteur (l'anatomiste 
Auguste Serres) tire d'un ouvrage s'appuyant presque entièrement sur l'observation de cas individuels, en dépit de la guerre des nombres franco-anglaise à laquelle il est censé contribuer ${ }^{1}$. Les conclusions auxquelles parviennent Alphonse Devergie (1851, 202-203) et Alexandre Brierre de Boismont (1865 [1856], 39-43, 493) d'après le dépouillement des dossiers de quelques milliers de suicidés parisiens sont moins vagues bien que sans valeur non plus. Les célibataires (comptés par Brierre de Boismont avec les veufs) se tueraient davantage que les époux. Il faut en réalité comprendre: parmi les 1223 suicidés reçus à la morgue entre 1837 et 1846 (Devergie), les célibataires devancent les époux, etc. GustaveFrançois Étoc-Demazy (1844, 72-73), lui, rapporte au total de la population de l'arrondissement du Mans les 87 suicides qui s'y produisent en 1830-1831 et découvre que les époux, dépassés toutefois par les veufs, se tuent plus que les célibataires. Il va jusqu'à se demander si l'état de mariage ne s'avère pas dangereux du point de vue du suicide (ibid., 197-198).

Le fait est qu'on parvient à des résultats incertains, voire contradictoires, même sur la base des séries administratives complètes des années 60 et suivantes. Autrement dit, on dispose finalement d'une assise de données adéquates, mais on a du mal à en dégager les régularités si longtemps cherchées. Les hommes célibataires restent à une nette distance des veufs dans le classement des individus les plus voués à la mort volontaire, mais l'emportent dans Le suicide d'Émile Durkheim (1897), point d'aboutissement de la sociologie du suicide à la fin du siècle. Quant à l'exposition des femmes au suicide, aucun sujet n'est plus controversé. À quoi il faut ajouter l'effet de complication engendré par l'introduction dans l'analyse des suicidés séparés de corps et, depuis 1884, divorcés.

Dans ce texte je poursuis trois objectifs, l'un principal, les deux autres secondaires. L'objectif premier est de restituer dans ses grandes lignes le débat sur les relations entre état civil et suicide jusqu'à Durkheim. Les objectifs secondaires sont les suivants: i) suggérer que, dans le cas français et en général, les statistiques officielles (la façon dont elles sont construites et leur fiabilité) ne sont pas les seules responsables des hésitations de la sociologie du suicide, la part devant être faite aux procédés à travers lesquels les données sont analysées et au mouvement des suicides eux-mêmes bien sûr; ii) distinguer dans Le suicide ce qui est inédit de ce qui est connu (déjà certifié par d'autres) relativement à un thème dont Philippe Besnard a montré l'intérêt à maintes reprises (1973; 1987, 70-98; 1997, 735-737). La prise en compte de l'état civil des suicidés relie en effet les chapitres (II-III et V) du livre II du Suicide traitant des deux types de suicide majeurs parmi les trois (quatre) identifiés. Mais où chercher les nouveautés dans ces chapitres? Certes pas au niveau des explications proposées, bien que personne avant Durkheim n'ait parlé d'intégration sociale et d'anomie. C'est vers les faits produits par Durkheim et les méthodes avec lesquelles il les établit qu'il faut se tourner. Les explications du Suicide ne sont évidemment rien si les faits sur lesquels elles s'appuient ne se tiennent pas et, c'est encore Besnard qui l'a fait voir, ces faits se prêtent parfois à

La guerre, visant à établir s'il y a plus de suicides à Paris ou à Londres, oppose Jean-Étienne Esquirol, dont Falret est un élève, à George M. Burrows de 1814 à 1822. Sa chronologie est dressée par Esquirol lui-même (1838, 1, 669-676). Ian Hacking (1990, 64) y a vu l'un des débuts de la sociologie quantitative. 
être interprétés différemment de ce qu'a fait Durkheim à condition qu'ils soient vrais. Il n'y a donc qu'à suivre cette piste.

\section{MARIAGE ET FAMILLE}

Arrivé au tableau XIV - et dernier - de son article, Bertillon père $(1872,50)$ aligne des chiffres révélateurs. Années 1863-1868, population au-dessus de 16 ans, taux moyens: en ce qui concerne les hommes (dont la sursuicidité est désormais acquise), le taux des veufs est plus du double de celui des célibataires qui se tuent plus que les hommes mariés $(628>273>246)$. En ce qui concerne les femmes, les veuves se tuent presque deux fois que les célibataires qui, cependant, se tuent moins que les femmes mariées $(133>59<62)$. I. e.: l'aptitude des gens mariés au suicide étant 100, celle du célibataire est 111 mais celle de la célibataire descend à 95. Or, Bertillon glisse sur le danger de suicide qui pèse sur la femme par le seul fait du mariage ainsi que sur le moindre danger (moins visible, il faut le reconnaître) qu'elle encourt dans le veuvage $(+255 \%$ pour les hommes, $+214 \%$ pour les femmes). Il s'arrête en revanche sur l'homme en développant l'argument selon lequel, comme le suicide croît avec l'âge, l'action de l'âge sur la tendance au suicide cache l'aggravation véritable qui vient à l'homme du célibat et du veuvage. Il propose de l'évaluer en calculant quel serait le taux de suicide des trois catégories d'état civil à leur âge moyen (données du recensement) si l'action de l'âge était la seule qui se fît sentir et trouve que l'influence du mariage sur la tendance au suicide va réduire de moitié celle de l'âge tandis que l'influence du veuvage l'aggrave. Après quoi il affirme que la même chose est valable pour les femmes: «Ainsi les deux sexes sont poussés au suicide par le fait du célibat et par celui du veuvage» (ibid., 52).

La plus grande suicidité de la femme mariée par rapport à l'homme relativement aux célibataires serait compensée par la maternité. 70\% des hommes mariés qui se suicident ont des enfants; sur 100 épouses, les mères sont au nombre de 61 . D'où la conclusion de Bertillon: «il est donc manifeste que la présence des enfants rattache plus à la vie la mère que le père » (ibid.).

Bertillon conçoit cette étude comme un prolongement de celle de la tendance au crime qu'il donne dans le même article pour les années 1840-1845 et 18611868. Il montre que parmi les gens mariés il y a moins d'accusés que parmi les célibataires et les veufs et veuves et que la femme mariée avec des enfants commet moins de crimes que son conjoint. Mais quelle est la différence par rapport à l'exposition de la femme au suicide par le seul fait du mariage? Elle réside en ceci, qu'en soi le mariage éloigne du crime la femme bien plus que l'homme (ibid., 35), la conclusion à en tirer (mais que Bertillon ne tire pas) étant alors que la même institution qui profite à la femme du point de vue du crime lui nuit du point de vue du suicide. En passant, Bertillon appelle «degré de préservation du crime par le fait du mariage » le chiffre que l'on obtient en divisant le taux de criminalité des célibataires par celui des époux ou épouses (ibid.). Cet indice donne de façon simplifiée les mêmes valeurs que les nombres relatifs habituels, mais Bertillon l'abandonne par la suite.

Les analyses de Bertillon sont tributaires de leur contexte. Ce statisticien s'applique à confirmer la thèse, qui date de 1858 (Farr, 1885, 438-440) et qui est en 
train de se propager ( $c f$. p. ex. Ébrard, 1870, 228), d'après laquelle l'«association conjugale» améliore la vie quel que soit l'aspect sous lequel on l'envisage et l'allonge aussi, à une exception près selon Bertillon: les mariages précoces (15-20 ans) qui (années 1856-1865, ibid., 46) affligent les jeunes époux d'une mortalité inconnue des célibataires du même âge - et à une seconde exception près involontairement occultée: le penchant au suicide éveillé chez la femme toutes choses égales par ailleurs. Une polémique s'engage entre Bertillon et Herbert Spencer. Ce dernier $(1873,92-96)$ ayant soutenu que le mariage est dépourvu de vertus propres et que l'explication des faits signalés par Bertillon également est autre, à savoir qu'une même prédisposition porte les plus aptes, à la fois, au mariage et à une vie sobre et longue, Bertillon $(1875 a, 770 ; 1875 b)$ lui répond que, s'il en était ainsi, on ne verrait pas les taux des veufs se rapprocher de ceux des célibataires ${ }^{2}$.

C'est dans un aperçu démographique de la France, rédigé encore pour le Dechambre, que Bertillon (1879, 473-474) revient sur l'effet de la présence d'enfants sur l'aptitude au suicide pour faire voir, en présentant cette fois des taux par million d'habitants pour les années 1861-1868, que cet effet s'étend aux veufs en sauvegardant toujours plus la mère que le père. Le danger de suicide est triplé chez la femme mariée sans enfants et plus que doublé chez la veuve stérile. Il double chez l'époux et le veuf sans enfants. Ces données, peu utilisables faute de rapport aux données des célibataires, sont mystérieuses quant à leurs sources. Durkheim lui-même $(1897,208)$, lorsqu'il les rencontre dans un des articles de Jacques Bertillon que je vais citer, les juge suspectes. Reste que le pas en avant accompli ici par Louis-Adolphe Bertillon est surtout dans le lexique, car les enfants, ce n'est pas le mariage mais c'est la famille, et le bon mot figure en effet: «cette salutaire influence de la famille se poursuit [...] après la mort de l'un des deux époux » (ibid., 474, c'est moi qui souligne).

Jacques Bertillon reprend et vulgarise les thèses de son père à quatre reprises au moins $(1879 a, b ; 1880 a, b)$ dans les textes préparatoires de sa grande enquête sur le divorce et la séparation de corps en Europe (1882). Un graphique représentant le rapport du suicide des gens mariés et des veufs à la présence d'enfants d'après les données de son père relatives à 1861-1868 a tout de suite pour titre: «Influence de la famille sur la tendance au suicide» $(1879 a, 782 ; 1879 b, 618)$. La distinction entre mariage (le couple) et famille (l'interaction du couple avec et par les enfants) est ainsi posée. Les deux institutions n'exercent pas la même action sur le suicide. Il s'agit de la tester empiriquement.

\section{FEMMES MARIÉES ET VEUVES}

Ce que Jacques Bertillon, précédé de Maurice Block $(1878,503)$, ne relève pas dans le tableau XIV de l'article de 1872 de son père est l'aggravation de la suicidité de la femme qui tient en propre au mariage et l'amélioration, bien relative, qu'elle reçoit du veuvage. Cela prouve que l'on ne voit que ce que l'on cherche. Il suffit de remarquer que Jacques Bertillon signale que la veuve met plus de temps

Spencer ne connaît l'article de Bertillon que d'après un compte rendu paru dans une gazette médicale. Bertillon de son côté lit The study of sociology dans la traduction de 1874 (Introduction à la science sociale, Paris, Guillaumin). 
à se remarier que le veuf du même âge $(1879 a, 778)$, ce qui veut bien dire que le mariage n'a pas les mêmes implications pour les deux sexes.

L'omission de Bertillon fils prouve aussi qu'il a lu d'une manière sélective $I l$ suicidio d'Enrico Morselli (1879), livre qui s'étend beaucoup sur le suicide en France et qu'il analyse dans sa revue, les Annales de démographie internationale, en survolant néanmoins le chapitre qui porte sur l'état civil des suicidés: «M. Morselli a bien voulu rappeler ici les travaux de mon père» ainsi que «un travail que j'ai fait sur le mariage », et c'est pratiquement tout (1879c, 637-638). Or, il est vrai que Morselli emprunte aux Bertillon des choses, dont la méthode pour évaluer l'influence du mariage sur l'action de l'âge en matière de suicidité (1879, 347-348), mais il est vrai aussi qu'à l'aide de données comparatives il: i) réalise sur-le-champ que la femme mariée se suicide plus que la célibataire et la veuve moins que le veuf; ii) vérifie, et donc ne se borne pas à répéter, que la présence d'enfants (la famille) protège davantage la femme que l'homme, bien que la vérification soit encore faite moyennant des données proportionnelles (sur 100 époux suicidés, combien d'hommes et de femmes sans enfants?).

C'est la comparaison avec l'Italie qui permet à Morselli de faire ses deux déductions concernant le suicide de la femme mariée et de la veuve. Dans ce pays (années 1873-1878), en égalant à 100 le taux de suicide des hommes mariés, celui des célibataires est de 120 et celui des veufs 235; en égalant à 100 le taux des femmes mariées, celui des célibataires est de 90 et celui des veuves 147 (ibid., 344). Ces rapports ne diffèrent des rapports français établis par Bertillon père que pour ce qui est du veuvage. Il y a davantage de veufs et surtout de veuves suicidés en France qu'en Italie. Cela ne change rien cependant quant à l'évidence d'un «résultat inattendu» que Morselli présente de la manière suivante: «le veuvage accroît le danger de suicide plus chez les hommes que chez les femmes et le célibat, qui nuit aux premiers, ne nuit pas autant aux secondes » (ibid.).

C'est, en revanche, la comparaison avec la Prusse (années 1869-1875) qui permet à Morselli de vérifier l'effet protecteur de la famille sur la femme. Ce pays est le seul dont la statistique publie des données pouvant être rapprochées des données françaises sur la présence d'enfants selon l'état civil. Les données de la statistique criminelle française ayant été mises à jour à 1867-1876 par l'auteur du Suicidio, il n'y a pas de surprises: «la présence d'enfants freine davantage la mère que le père dans le mariage et dans le veuvage» (ibid., 350). La logique semble mener toute seule à la conclusion à laquelle Morselli essaie d'arriver par approximation en reprenant de Bertillon père le calcul du nombre de fois où, en France, la femme stérile se suicide plus que la mère dans le mariage et dans le veuvage par rapport à l'homme: si le célibat est moins dangereux pour la femme que pour l'homme et si la mère se tue moins que son mari, l'aggravation de la tendance de la femme au suicide qui tient en propre au mariage doit être à la charge de la femme sans enfants dans une mesure non négligeable. D'autre part, si le veuvage profite à la femme par le seul fait qu'il se produit - qu'elle ait des enfants ou pas -, cela veut dire que le mariage n'est pas en soi et de toute façon la condition la meilleure pour la femme du point de vue du suicide.

Quatre remarques de Morselli sont à retenir. La première est que partout en Europe ce sont les veufs qui paient le tribut le plus élevé à la mort volontaire, hormis les pays où le divorce existe. Là où le divorce est admis (Prusse, Saxe, Würtemberg, etc.), la palme revient aux divorcés (ibid., 343 et 346). Adolph 
Wagner $(1864,179)$ avait déjà signalé ce fait. La seconde remarque est que l'homme divorcé profiterait de la présence d'enfants plus que la femme, à la différence de ce qui se passe dans le veuvage. Morselli ne dispose toutefois que des faibles données de la Prusse, le seul endroit où l'on peut comparer les taux des veufs et des divorcés à cette date $(1879,350)$. Cette remarque est complémentaire de la troisième, selon laquelle, comme on devient veuf à mesure que l'on vieillit mais qu'on divorce à tous les âges et de préférence jeune, le suicide des veufs et celui des divorcés ne relèvent pas de la même cause (ibid., 347). Enfin Morselli souscrit à une thèse qu'il trouve chez Jean-Baptiste Cazauvieilh $(1840,253)$ et qui est décidément minoritaire ( $c f$. Lisle, 1856, 33-34), à savoir que les femmes, sans autres précisions, se tueraient moins dans les villes que dans les campagnes par rapport aux hommes. Entre 1866 et 1870 les suicides féminins représenteraient $20,8 \%$ des suicides dans les centres de moins de 2000 habitants et 18,5\% dans les autres, et ainsi de suite (1879, 304-305).

La parution du livre de Morselli prend à contre-pied Alfred Legoyt qui prépare son Suicide ancien et moderne (1881), axé lui aussi, dans sa seconde partie, sur des comparaisons internationales. Qu'ajouter à ce qu'a dit l'aliéniste italien au sujet des relations entre état civil et suicide? Deux choses: il semble vraiment que la femme soit pénalisée du point de vue du suicide par le fait du mariage en France et en Italie seulement (mais là Legoyt se contente de retravailler les statistiques de Morselli); s'il est question de la France, ce désavantage peut être confirmé à la lumière de données plus récentes que celles de Bertillon père, quoique moins parlantes. Année 1876 (les garçons de moins de 18 ans et les filles de moins de 15 ans étant soustraits): les veufs se tuent plus que les célibataires qui se tuent plus que les hommes mariés $(737>422>272)$; les veuves se tuent plus que les célibataires qui toutefois se tuent légèrement moins que (ou presque comme) les femmes mariées $(121>80<81)$. C'est-à-dire que le taux des hommes célibataires est à celui des hommes mariés comme 155 est à 100 tandis que pour 100 femmes mariées suicidées il y a 99 femmes célibataires. On voit alors que l'influence bienfaisante du mariage sur la tendance au suicide - influence qui est désormais à l'ordre du jour ${ }^{3}$ - est démontrée, «mais en ce qui concerne les hommes seulement $\gg\left(\right.$ ibid., $\left.169-172^{4}\right)$.

\section{LE SUICIDE DE LA FEMME MARIÉE S'ÉCLIPSE}

Un tournant dans la sociologie française du suicide se produit en 1882. Jacques Bertillon publie sa recherche sur le divorce et Émile Yvernès, chef de la statistique judiciaire, fait paraître (de manière anonyme, selon les usages du Ministère de la justice) le volume du Compte pour l'année 1880 destiné à devenir une référence en raison des tableaux récapitulatifs (à partir de 1826) qu'il contient. Bertillon fils met en évidence que la «relation imprévue» entre le divorce et le suicide qu'il observe en Europe et qui consiste dans le fait que les deux phénomènes varient ensemble, fonctionne en France aussi si on raisonne sur

Voir Decaisne, 1880, 122; Motet, 1880, 103; Chaussinand, 1881, 86.

Cf. également Legoyt, 1884, 258-260. 
les séparations de corps: les départements où il y a le plus de séparations (combien en moyenne pour 1000 mariages de 1860 à 1879?) sont les mêmes que ceux où il y a le plus de suicides (taux moyen de 1872 à 1876) (1882, 303-304 et 337-338). Cette donnée est aussi remarquable qu'elle est peu utilisable: comment comparer le taux des séparés à celui des veufs et des célibataires?

Yvernès, quant à lui, actualise les comptes à 1876-1880. En regroupant les époux suicidés avec les veufs, il montre que $65 \%$ des hommes et $59 \%$ des femmes ont des enfants. L'effet protecteur assuré par la famille au profit de la femme continue somme toute dans la mesure établie par Bertillon père. Il n'en va pas de même si l'on regarde l'action du mariage. En ce qui concerne les hommes (valeurs moyennes arrondies, hormis les mineurs là aussi), le taux des veufs dépasse celui des célibataires qui est double de celui des époux $(760>490$ $>290$ ). En ce qui concerne les femmes par contre, le taux des célibataires, tout en restant d'un tiers au-dessous de celui des veuves relativement à 1876 (données de Legoyt), devance nettement maintenant le taux des épouses $(150>100>80)$ (Yvernès, 1882, CXV). On ne voit plus l'aggravation de la tendance de la femme au suicide qui tient en propre au mariage et elle disparaît, en effet, de l'horizon des études sur le suicide ( $c f$. p. ex. Levasseur, 1889-1892, 2, 128) exception faite pour les auteurs qui s'en tiennent aux anciennes données (Morselli, 1885, 299; Corre, 1891, 472).

On ne voit plus cette aggravation quoiqu'elle existe encore. Elle est bel et bien dans le fait que, dans le mariage, la femme demeure plus proche de la célibataire du point de vue du suicide que ne l'est l'homme par rapport au célibataire. Chiffres d'Yvernès: les hommes célibataires sont aux époux comme 169 est à 100 ; pour les femmes célibataires le rapport aux épouses est de 125 à 100. Cependant une redistribution du poids du suicide entre les groupes d'état civil est en cours en France au sein de la hausse générale de la mort volontaire dont tout le monde se plaint et dont il n'est pas permis de douter (Chesnais, 1976, 41-43, 305). Proche de la célibataire de par son aptitude au suicide, la femme mariée l'est de moins en moins. Cela contribue à expliquer que son taux, qui dans les années 60 dépassait celui de la célibataire, lui est maintenant inférieur.

\section{LES HOMMES CÉLIBATAIRES EN TÊTE}

L'influence de l'état civil sur la tendance au suicide ne pouvant être dégagée comme il faut de l'influence de l'âge que si l'on parvient à comparer les taux de gens du même âge, Durkheim se résout à faire faire à Marcel Mauss, comme on le sait, le dépouillement de trois années (1889-1991) de dossiers de suicides (environ 25500) à la recherche de l'information qui manque: à la fois l'état civil et l'âge pour chaque suicidé des deux sexes. Et ce entre la fin 1895 et le début 1896. Dans le tableau XXI de son livre $(1897,183)$, il présente les données ainsi obtenues en les regroupant par tranches d'âge et compare les taux au moyen d'un «coefficient de préservation» qui n'est autre, de par la manière dont il est composé, que le «degré de préservation» (du crime) qu'il trouve chez Bertillon père (voir supra). Plus ce coefficient est élevé et plus la catégorie d'état civil que l'on considère est protégée (= elle se tue moins) par rapport à celle dont le taux figure au numérateur. $\mathrm{Au}$-dessous de 1, le coefficient mesure une aggravation. 
Si dans le tableau de Durkheim on calcule les coefficients de préservation sur la base des taux moyens (ce qui diffère de ce que fait Durkheim qui utilise régulièrement la moyenne des taux, les deux procédés ne donnant néanmoins pas ici de résultats contraires) et si l'on traduit en coefficients les nombres relatifs de Bertillon père, Legoyt et Yvernès (des nombres qui, rappelons-le, sont à leur tour établis à partir de taux moyens ${ }^{5}$ ), on obtient que les données de Durkheim peuvent être rapprochées de celles de ses devanciers français de la façon montrée dans le tableau ci-après. En admettant que ces quatre auteurs soient fidèles aux données du Ministère de la Justice (qu'elles soient publiées ou inédites) et que l'écart entre la suicidité apparente enregistrée par ces données et la réelle soit constante au fil des ans, ce tableau résume aussi l'évolution du suicide en France de 1863 à 1891.

France. Suicides commis par million d' habitants de chaque groupe d'état civil à quatre époques différentes selon quatre auteurs

\begin{tabular}{|c|c|c|c|c|c|c|c|}
\hline & \multirow[b]{3}{*}{ Veufs } & \multirow[b]{3}{*}{ Célibataires } & \multirow[b]{3}{*}{ Époux } & \multicolumn{3}{|c|}{$\begin{array}{c}\text { COEFFICIENTS DE PRÉSERVATION } \\
\text { DES }\end{array}$} \\
\hline & & & & & \multicolumn{2}{|c|}{ Époux } & \multirow{2}{*}{$\begin{array}{c}\text { Veufs } \\
\text { Par rapport } \\
\text { aux } \\
\text { célibataires }\end{array}$} \\
\hline & & & & & $\begin{array}{l}\text { Par rapport } \\
\text { aux } \\
\text { célibataires }\end{array}$ & \begin{tabular}{|c|} 
Par rapport \\
aux \\
veufs
\end{tabular} & \\
\hline \multicolumn{2}{|c|}{ L.-A. BERTILLON (1872) } & & & & & & \\
\hline 1863-1868 & $\mathrm{H}$ & $\begin{array}{l}628 \\
133\end{array}$ & $\begin{array}{l}273 \\
59\end{array}$ & $\begin{array}{c}246 \\
62\end{array}$ & $\begin{array}{l}1,11 \\
0,95\end{array}$ & $\begin{array}{l}2,55 \\
2,14\end{array}$ & $\begin{array}{l}0,43 \\
0,44\end{array}$ \\
\hline \multicolumn{2}{|c|}{ A. LEGOYT (1881) } & & & & & & \\
\hline 1876 & $\begin{array}{l}\mathrm{H} \\
\mathrm{F}\end{array}$ & $\begin{array}{l}737 \\
121\end{array}$ & $\begin{array}{c}422 \\
80\end{array}$ & $\begin{array}{l}272 \\
81\end{array}$ & $\begin{array}{l}1,55 \\
0,99\end{array}$ & $\begin{array}{l}2,71 \\
1,49\end{array}$ & $\begin{array}{l}0,57 \\
0,66\end{array}$ \\
\hline \multicolumn{2}{|c|}{ É. YVERNÈS (1882) } & & & & & & \\
\hline $1876-1880$ & $\begin{array}{l}\mathrm{H} \\
\mathrm{F}\end{array}$ & $\begin{array}{l}760 \\
150\end{array}$ & $\begin{array}{l}490 \\
100\end{array}$ & $\begin{array}{c}290 \\
80\end{array}$ & $\begin{array}{l}1,69 \\
1,25\end{array}$ & $\begin{array}{l}2,62 \\
1,87\end{array}$ & $\begin{array}{l}0,64 \\
0,67\end{array}$ \\
\hline \multicolumn{2}{|c|}{ É. DURKHEIM (1897) } & & & & & & \\
\hline $1889-1891$ & $\begin{array}{l}\mathrm{H} \\
\mathrm{F}\end{array}$ & $\begin{array}{l}713 \\
210\end{array}$ & $\begin{array}{l}1011 \\
156\end{array}$ & $\begin{array}{l}435 \\
108\end{array}$ & $\begin{array}{l}2,32 \\
1,44\end{array}$ & $\begin{array}{l}1,64 \\
1,94\end{array}$ & $\begin{array}{l}1,42 \\
0,74\end{array}$ \\
\hline
\end{tabular}

On voit dans ce tableau la surprise à laquelle Durkheim est confronté. Pour la première fois le taux des hommes célibataires dépasse (et de presque un tiers) celui des veufs, l'écart entre ce dernier taux et celui des hommes mariés n'ayant jamais été par ailleurs si petit. Ces modifications, qui prennent place dans la montée du suicide masculin qui commence vers 1886 et a son pic entre 1891 et 1895 (Chesnais, 1976, 42-44), entraînent une conséquence également insolite: l'homme paraît plus protégé que la femme dans le veuvage, par rapport aux gens mariés et, nettement, par rapport aux célibataires. Comment rendre compte de ces faits? Durkheim ne considère pas le moins du monde que l'explication est banale, à savoir que le mouvement des suicides se retourne (parfois) contre les unifor-

\footnotetext{
La transformation des nombres relatifs en coefficients se fait rapidement: il suffit de mettre la
} virgule avant les deux derniers chiffres. 
mités statistiques dans lesquelles on essaie de le brider, et, en intégrant à ses données celles de la seule statistique exploitable qui affiche simultanément l'état civil et l'âge des suicidés, la statistique du Grand-Duché d'Oldenbourg pour les années 1871-1885, se consacre à la poursuite des «lois » capables de faire rentrer dans la norme ce qui semble une anomalie d'après les conclusions d'un demisiècle de statistique morale. En d'autres termes: des Bertillon à Yvernès, en passant par Morselli, on s'est trompé.

La détermination de la part que le mariage (la «société conjugale») et la famille («la société domestique» ou «société familiale») prennent respectivement dans la protection vis-à-vis du suicide (le but que Durkheim s'était fixé en décidant d'examiner ces dossiers) se croise ainsi dans le chapitre III du livre II du Suicide avec la réfutation du «préjugé qui fait du veuvage la plus disgraciée de toutes les conditions au point de vue du suicide» (ibid., 178). Les deux arguments peuvent cependant être séparés.

\section{À QUOI TIENT L'IMMUNITÉ DES GENS MARIÉS?}

En ce qui concerne les effets combinés du mariage et de la famille sur l'aptitude au suicide, il va presque de soi que le coefficient de préservation des gens mariés est supérieur à celui des célibataires du même âge et que, en France, celui des époux dépasse celui des épouses. Il est beaucoup moins manifeste que cela ne se vérifie qu'au-dessus de 20 ans. Et pourtant il en est ainsi : à Oldenbourg aussi les jeunes époux, notamment les hommes, se suicident plus que les célibataires. Cette donnée va renforcer les conjectures de Bertillon père sur les tendances de la mortalité générale aux différents âges et est confirmé par une statistique suédoise ( $c f$. Bertillon J., 1879b, 619), citée par Durkheim (1897, 179-181) bien que ce ne soit pas à ce propos. C'est la première fois que le phénomène du suicide des jeunes époux est mis en évidence en France.

Durkheim compare ensuite les taux des époux et des veufs français avec et sans enfants de 1887 à 1891 (valeurs moyennes) - taux calculé par rapport au nombre des ménages avec et sans enfants enregistré par le recensement de 1886 qui, comme je l'ai dit au début, ne donne pas la distribution selon l'âge - aux taux des célibataires de la tranche d'âge correspondant à l'âge moyen de ces quatre groupes (ses propres données de 1889-1891). Il parvient ainsi à établir les faits qu'il condense dans le tableau non numéroté de la page $207^{\circ}$. Ces faits se prêtent à être exposés de la façon suivante:

1/ Les coefficients des hommes mariés sans enfants et des veufs sans enfants sont assez proches $(1,5$ et 1,2$)$ et il en va de même pour ceux des épouses et des veuves stériles $(0,67$ et 0,60$)$. S'il était vrai que la protection contre le suicide vient du mariage, les coefficients des époux devraient être bien supérieurs à ceux des veufs. 2/ Les époux et les veufs avec enfants $(2,9$ et 1,6) et les épouses et les veuves avec enfants $(1,89$ et 1,06$)$ ont les coefficients les plus élevés. S'il était vrai que c'est le mariage qui protège, le coefficient des veufs et des veuves ne devrait

Familier aux spécialistes de Durkheim depuis les variations que Besnard (1973, 46-52) y a introduit. 
pas monter. 3/ Les coefficients des épouses et des veuves stériles marquent en réalité une aggravation par rapport aux femmes célibataires. Cela veut dire que le mariage ne sauvegarde aucunement la femme, à la différence de ce qui se passe pour l'homme qui, même sans enfants $(1,5)$, y trouve un abri.

Deux conséquences découlent des faits ainsi définis. La première est qu'on n'est réellement protégé contre le suicide que par la présence d'enfants, l'immunité des gens mariés étant due «toute entière pour un sexe [les femmes] et en majeure partie pour l'autre, à l'action, non de la société conjugale, mais de la société familiale» $(1897,197)$. La seconde conséquence est que l'aggravation du penchant de la femme au suicide qui tient au mariage n'est redevable à l'absence d'enfants que d'une façon marginale, la stérilité jouant ici le rôle d'un facteur qui agit sur un terrain favorable. Ce terrain est que «par elle-même, la société conjugale nuit à la femme» du point de vue du suicide (ibid., 196), comme cela résulte aussi du fait que, à chaque tranche d'âge, la part que les femmes prennent au suicide des gens mariés est supérieure à celle qu'elles prennent au suicide des gens célibataires (ibid., 190).

Les progrès par rapport à la sociologie du suicide précédente sont au nombre de deux également: les effets du mariage sur le suicide s'avèrent au total plus morbides que prophylactiques étant donné que même la protection dont jouit l'homme dans le mariage (Morselli et Legoyt) ne suffit pas à le sauvegarder complètement du danger du suicide; le cas du suicide de la femme mariée sans enfants envisagé par Morselli n'existe pas ${ }^{7}$. Il existe le cas du suicide de la femme mariée tout court qu'on peut mettre sous cette forme: ce suicide a dans la présence d'enfants son seul frein, mais n'a pas dans l'absence d'enfants sa cause véritable. Il demeure par contre correct d'affirmer que la présence d'enfants protège plus la femme que l'homme (Bertillon père, Morselli et Yvernès) même si les coefficients des hommes avec enfants sont supérieurs à ceux des femmes de même condition en particulier dans le mariage $(2,9>1,89)$. C'est que les bénéfices que la femme retire de la maternité ne suffisent pas à annuler le désavantage qui lui est procuré par le mariage relativement à l'homme.

La découverte qu'il n'y a que la famille qui préserve totalement du suicide permet à Durkheim de retrouver sa thèse de 1888 selon laquelle le suicide régresse à mesure que la natalité augmente et de la préciser en faisant voir que la carte du suicide en France se superpose parfaitement à celle de la densité des ménages (ibid., 210-212).

De son côté, le fait que le mariage nuit à la femme du point de vue du suicide ne se tient que si on pose que «la vie de famille [en raison des effets qu'y produit l'action du mariage justement] affecte différemment la constitution morale des deux sexes» (ibid., 190). Durkheim a recours à ce thème pour compléter la critique de Bertillon père à l'idée que les privilèges du mariage (par rapport au célibat) proviendraient de la sélection que cette institution opère entre les candidats (les célibataires eux-mêmes). S'il en était ainsi, note-t-il, les époux et les épouses devraient se tuer avec la même fréquence. Au contraire, la femme mariée est moins immunisée que l'homme et sa moindre immunité grandit avec l'âge,

Malgré la dernière note de bas de page du chap. V du livre II du Suicide (la note sur le suicide fataliste) qui aura pour résultat de gonfler ce cas dans les études durkheimiennes. 
c'est-à-dire à mesure qu'elle est exposée aux effets du mariage (ibid., 191). Phénomène tout d'abord français, cette constitution du mariage, si «désastreuse pour la femme» du point de vue du suicide (ibid., 201), est-elle généralisable? La question est justifiée, car à Oldenbourg, seul terme de comparaison admis, le sexe le plus favorisé dans le mariage est la femme (ibid., 185 et 191).

Finalement, en raisonnant encore sur le fait que le père perd beaucoup dans le veuvage (de 2,9 à 1,6) tandis que l'époux sans enfants y perd moins (de 1,5 à 1,2), Durkheim croit repérer la cause du suicide du veuf dans la désorganisation de la famille découlant de la mort de la mère et non dans la dissolution du mariage (mort de l'épouse) (ibid., 195-196 et 290).

\section{QUAND LE LIEN CONJUGAL S'AFFAIBLIT}

Ce sont encore les données du Grand-Duché d'Oldenbourg qui aident Durkheim à contourner l'évidence du fait que deux événements exceptionnels concomitants, une augmentation de plus de $100 \%$ du suicide des hommes célibataires par rapport à la période 1876-1880 et une baisse même surprenante des suicides des veufs (descendu au-dessous du niveau de 1876), sont à l'origine de la plus grande immunité dont semblent jouir les veufs relativement aux veuves en France pendant les années 1889-1891. Il se trouve en effet qu'à Oldenbourg non seulement le mariage, mais aussi le veuvage profite davantage à la femme qu'à l'homme. Comme en France c'est l'inverse, Durkheim en conclut à l'existence d'une loi: dans une même société, la tendance au suicide de chaque sexe à l'état de veuvage prolonge celle de ce même sexe à l'état de mariage (ibid., 203 et 205).

Deux cas seulement ne suffisant pas à soutenir une telle proposition, Durkheim isole le département de la Seine et calcule, séparément pour l'agglomération parisienne et la province, les taux de suicide des gens mariés et des veufs ainsi que leurs coefficients de préservation relativement aux célibataires. Il établit de la sorte que, en province, où les époux sont plus protégés que les épouses, les veufs se tuent moins que les veuves, tandis que dans la Seine, où les épouses sont les plus préservées, les veufs se tuent plus que les veuves. Au passage, Durkheim croit prendre en faute Morselli qui aurait exagéré la contribution de la femme aux suicides accomplis par les deux sexes à l'état de veuvage en oubliant qu'il y a partout plus de veuves que de veufs (ibid., 200).

Morselli n'a pas commis cette erreur ${ }^{8}$ et il est fortement douteux que le rapprochement de la France urbaine de la France rurale proposé par Durkheim soit utile à l'étude des conséquences du veuvage sur l'aptitude au suicide. En comparant directement (c'est-à-dire sans passer par les célibataires) les taux des gens mariés à ceux des veufs d'après les données du Suicide, Besnard (1973, 52-54) a obtenu un résultat contraire à celui de Durkheim: l'homme est plus pénalisé que la femme dans le veuvage aussi bien dans la Seine qu'en province. Il est vrai que le coeffi-

Il a consciemment raisonné sur des chiffres absolus dans les pages que Durkheim prend pour cible (cf. Morselli, 1879, 340-342). Même Maurice Halbwachs $(1930,198)$ prête cette erreur à Morselli sur la base, lui, d'une véritable erreur de traduction; ce n'est pas clair s'il s'agit de l'italien à l'allemand ou de l'allemand au français. Halbwachs utilise en effet l'édition allemande du livre de Morselli (1881). 
cient de préservation des époux par rapport aux veufs ne mesure pas la même chose que celui des veufs par rapport au célibataires (l'anomalie des années 18891891 relativement aux décennies précédentes résidant surtout dans les valeurs de ce dernier coefficient), mais il y en a assez pour se défier de la loi d'après laquelle le penchant au suicide à l'état de veuvage suivrait celui à l'état de mariage.

En revanche, l'exposition différente des deux sexes au suicide dans le mariage selon le milieu est incontestable. D'où cela vient-il? Du fait que dans la Seine le lien conjugal est plus faible qu'en province. Or, le mariage étant en soi nocif pour la femme du point de vue du suicide, tout relâchement du mariage ne peut que lui profiter. Durkheim arrive à cette conclusion à travers une analyse particulière de la relation entre divorce et suicide, analyse qui lui permet de répondre à une seconde question: pourquoi, dans le mariage, l'immunité de la femme au suicide augmente-t-elle en même temps que celle de l'homme diminue, car c'est bien ce phénomène que l'on peut observer en France à mesure que l'on passe de la périphérie au centre?

L'originalité de l'analyse de la relation divorce-suicide mise en œuvre par Durkheim consiste en ceci: en donnant pour certaine la forte fréquence du suicide parmi les divorcés (elle est la plus élevée, données de quatre pays), Durkheim compare les taux des gens mariés à ceux des célibataires là où le divorce existe (Bade, Prusse et Saxe) et là où il n'existe pas (Italie et France ${ }^{9}$ ) et se demande si le fait que la rupture du mariage soit possible modifie l'action de ce dernier sur l'aptitude au suicide des deux sexes. Le renversement de la règle qui voit chez l'homme le seul bénéficiaire dans le mariage n'aurait d'autre cause que cette possibilité. Il suffit que la société conjugale soit ébranlée par la perspective d'un événement qui n'est pas nécessairement destiné à se réaliser pour que l'homme et la femme se retrouvent, d'un seul coup, respectivement plus et moins à la merci du suicide par rapport aux célibataires. Les données par lesquelles la province française et le département de la Seine entrent dans cette comparaison (données de 1885, ibid., 297) reflètent alors une tendance générale: la modernisation des sociétés, qui passe par les villes et entraîne une déstabilisation de l'institution du mariage (c'est enfin connu), profite à l'épouse et nuit à l'époux du point de vue du suicide (ce qui confirme les vues de Cazauvieilh et Morselli sur l'amélioration que la femme reçoit de la vie urbaine).

Durkheim, qui établit, d'après les données françaises permettant de comparer les taux des groupes d'état civil selon l'âge, l'existence - donc en France - de deux constitutions morales différentes à l'origine de l'inégale exposition au suicide de l'homme et de la femme dans le mariage, s'appuie ainsi sur des données européennes d'ensemble pour déceler la nature de ces constitutions et suggérer qu'on a affaire à un phénomène répandu. Déduites du comportement des deux sexes face à l'affaiblissement du lien conjugal qui se produit lorsque le divorce est possible, les constitutions morales de l'homme et de la femme sont les suivantes: "l'un a besoin de contrainte et l'autre de liberté», c'est-à-dire que «leurs intérêts sont contraires», voire « antagonistes» (ibid., 302 et 309).

Faute de la contrainte dérivant de l'indissolubilité du mariage, l'homme fait l'expérience de l'illimitation du désir sexuel. Il la partage avec le célibataire et

Données antérieures à 1884 bien sûr. 
c'est cette condition qui caractérise le suicide du divorcé par rapport à celui du veuf (la dissemblance entre ces deux types de suicide étant restée énigmatique pour Morselli, comme on se le rappellera). Suit un excursus sur les désirs sexuels de la femme, «naturellement bornés », qu'on ne saurait reproposer ici. Ce qu'il faut retenir de cette analyse est que, quelle que soit la façon dont on l'explique, la loi selon laquelle plus le divorce est pratiqué, plus la femme est préservée du suicide dans le mariage relativement à la célibataire (ibid., 302) - loi délaissée à un certain moment par Durkheim lui-même (1906) - était encore valable en France un siècle après sa formulation ( $c f$. Besnard, 1997).

Quoi de neuf donc dans Le suicide à propos de la relation entre état civil et mort volontaire? En ce qui concerne les faits établis, outre la découverte de l'influence aggravante des mariages précoces, la détermination de la part qui revient à la présence d'enfants dans l'immunité des deux sexes et l'établissement des deux uniformités empiriques de valeur inégale selon lesquelles, respectivement, le degré de préservation dont jouissent les deux sexes dans le mariage se prolonge dans le veuvage et le désavantage, pour la femme, et l'avantage, pour l'homme, dus au mariage se réduisent à mesure que cette institution perd de l'importance dans la société. En ce qui concerne les procédés par lesquels Durkheim parvient à ces même faits, presque tout est inédit dans la manière dont l'auteur du Suicide combine les données d'une recherche que personne n'a répétée depuis (celle sur les dossiers des suicidés) et ces mêmes données avec celles des statistiques officielles - hormis l'usage qu'il fait du coefficient de préservation emprunté à Louis-Adolphe Bertillon ${ }^{10}$.

Dipartimento di Scienze Sociali

Università di Torino

m.borlandi@virgilio.it

\section{RÉFÉRENCES BIBLIOGRAPHIQUES}

Bertillon L.-A., 1872, Mariage (démographie), in A. Dechambre (sous la dir. de), Dictionnaire encyclopédique des sciences médicales, Paris, Masson \& Asselin, $2^{\text {c }}$ s., 5, 7-52.

- 1875 a, Mortalité, in A. Dechambre (sous la dir. de), Dictionnaire encyclopédique des sciences médicales, Paris, Masson \& Asselin, $2^{\circ}$ s.,9, 725-791.

- $\quad 1875 b$, Une critique malheureuse de M. Herbert Spencer à propos du mariage et de son influence sur la vitalité, La philosophie positive, 15, 242-251.

- 1879, France (démographie), in A. Dechambre (sous la dir. de), Dictionnaire encyclopédique des sciences médicales, Paris, Masson \& Asselin, 4e s., 5, 403-584.

Bertillon J., 1879a, Les célibataires, les veufs et les divorcés au point de vue du mariage, La Revue scientifique, $2^{\mathrm{c}}$ s., 16, 776-783.

- $\quad 1879 b$, Note sur l'influence du mariage sur la tendance au suicide, Annales de démographie internationale, 3, 617-621.

1o Cet article s'appuie sur la bibliographie des études sur le suicide au XIX ${ }^{\mathrm{e}}$ siècle établie in Borlandi, 2000 et 2003. On se reportera aussi à Ronsin (1995) et au chapitre 8 de The taming of chance de Hacking dont il existe une précédente version française ( $c f$. Hacking, 1982 et 1990). La question de la fiabilité des statistiques du suicide que j'ai évoquée en deux occasions a été abordée, à propos du Suicide, par Besnard (1976) et Merllié (1987). 
- $\quad 1879 c$, Compte rendu de E. Morselli (1879), Annales de démographie internationale, 3, 622-642.

- $\quad 1880 a$, Note pour l'étude statistique du divorce, Annales de démographie internationale, 4, 457 480.

- $\quad 1880 b$, De l'influence du mariage sur la tendance au suicide, Bulletins de la Société d'anthropologie de Paris, $3^{\text {e }}$ s., 3, 277-286, 288-291.

- $\quad 1882$, Étude démographique du divorce et de la séparation de corps dans les différents pays de l'Europe, Annales de démographie internationale, 6, 257-364, 385-451.

Besnard Ph., 1973, Durkheim et les femmes ou Le suicide inachevé, Revue française de sociologie, 14 (1), 27-61.

- 1976, Anti- ou anté-durkheimisme? Contribution au débat sur les statistiques officielles du suicide, Revue française de sociologie, 17 (2), 313-341.

- 1987, L'anomie, ses usages et ses fonctions dans la discipline sociologique depuis Durkheim, Paris, Presses universitaires de France.

- $\quad$ 1997, Mariage et suicide: le théorie durkheimienne de la régulation à l'épreuve d'un siècle, Revue française de sociologie, 38 (4), 735-758.

Block M., 1878, Traité théorique et pratique de statistique, Paris, Guillaumin.

Borlandi M., 2000, Lire ce que Durkheim a lu. Enquête sur les sources statistiques et médicales du Suicide, in M. Borlandi et M. Cherkaoui (sous la dir. de), Le suicide un siècle après Durkheim, Paris, Presses universitaires de France, 9-46.

- 2003, De Morselli à Durkheim (et retour): contribution à l'histoire de la sociologie du suicide, in M. Cherkaoui (sous la dir. de), Histoire et théorie des sciences sociales, Genève-Paris, Droz, 33 55 .

Brierre de Boismont A., 1865[1856], Du suicide et de la folie suicide, Paris, Baillière.

Cazauvieilh J.-B., 1840, Du suicide, de l'aliénation mentale et des crimes contre les personnes, comparés dans leurs rapports réciproques, Paris, Baillière.

Chaussinand H., 1881, Étude de la statistique criminelle de la France au point de vue médico-légal, Lyon, Duc.

Chesnais J.-C., 1976, Les morts violentes en France depuis 1826, Paris, INED-Presses universitaires de France.

Corre A., 1891, Crime et suicide. Étiologie générale, facteurs individuels, sociologiques et cosmiques, Paris, Doin.

Decaisne E., 1880, Le suicide en France, Journal de la Société statistique de Paris, 21 (5), 121-125.

Devergie A., 1851, Statistique décennale de la Morgue, 1836-1846, Annales d' hygiène publique et de médecine légale, 45, 182-215.

Durkheim É., 1888, Suicide et natalité, Revue philosophique, 26, 446-463. [Repr. in Durkheim, 1975, 2, 216-236.]

- $\quad$ 1897, Le suicide. Étude de sociologie, Paris, Alcan.

- 1906, Le divorce par consentement mutuel, Revue bleue, 44 (5), 549-554. [Repr. in Durkheim, 1975, 2, 181-194.]

- 1975 , Textes, Paris, Minuit, 3 vol.

Ébrard N., 1870, Du suicide considéré aux points de vue médical, philosophique, religieux et social, Avignon, Seguin.

Esquirol J.-E.-D., 1838, Des maladies mentales, considérées sous les rapports médical, hygiénique et médico-légal, Paris, Baillière, 2 vol.

Étoc-Demazy G.-F., 1844, Recherches statistiques sur le suicide, appliquées à l' hygiène publique et à la médecine légale, Paris, Baillière.

Falret J.-P., 1822, De l'hypochondrie et du suicide. Considérations sur les causes, sur le siège et le traitement de ces maladies, Paris, Croullebois.

Farr W., 1885, Vital statistics: a memorial volume of selections from the reports and writings of William Farr, London, Stanford. 
Hacking I., 1982, La statistique du suicide au XIX ${ }^{\mathrm{e}}$ siècle, in A. Fagot (sous la dir. de), Médecine et probabilité, Créteil, Université de Paris-Val-de-Marne, 165-185.

- 1990, The taming of chance, Cambridge, Cambridge University Press.

Halbwachs M., 1930, Les causes du suicide, Paris, Alcan.

Levasseur É., 1889-1892, La population française, Paris, Rousseau, 3 vol.

Legoyt A., 1881, Le suicide ancien et moderne. Étude historique, philosophique, morale et statistique, Paris, Drouen.

- $\quad$ 1884, Suicide (\$ 1-5), in A. Dechambre (sous la dir. de), Dictionnaire encyclopédique des sciences médicales, Paris, Masson \& Asselin, $3^{\mathrm{e}}$ s., 13, 242-296.

Lisle E., 1856, Du suicide. Statistique, médecine, histoire et législation, Paris, Baillière.

Merllié D., 1987, Le suicide et ses statistiques: Durkheim et sa postérité, Revue philosophique, 112 (3), 303-325.

Morselli E., 1879, Il suicidio. Saggio di statistica morale comparata, Milano, Dumolard.

- 1881, Der Selbstmord. Ein Kapitel aus der Moralstatistik, Leipzig, Brockhaus.

- 1885, Le leggi statistiche del suicidio secondo gli ultimi documenti (1879-1885), Giornale della reale società italiana d'igiene, 7, 256-341.

Motet A.-A., 1880, Les morts accidentelles et les suicides en France, Annales d' hygiène publique et de médecine légale, $3^{\mathrm{c}} \mathrm{s} ., 3$, , 97-110.

Quetelet A., 1835, Sur l'homme et le développement de ses facultés ou Essai de physique sociale, Paris, Bachelier, 2 vol.

Ronsin F., 1995, Une aventure de jeunesse de la sociologie: les relations entre divorce et suicide, Revue d' histoire moderne et contemporaine, 42 (2), 292-312.

Spencer H., 1873, The study of sociology, London, King.

Wagner A. H. G., 1864, Die Gesetzmässigkeit in den scheinbar willkührlichen menschlichen Handlungen vom Standpunkte der Statistik, Hamburg, Boyes und Geisler, 2 vol. 